\title{
Solutions to Yang-Mills Equations on Four-Dimensional de Sitter Space
}

\author{
Tatiana A. Ivanova,,$^{1, *}$ Olaf Lechtenfeld,,$^{2,3, \dagger}$ and Alexander D. Popov ${ }^{2, \$}$ \\ ${ }^{1}$ Bogoliubov Laboratory of Theoretical Physics, JINR, 141980 Dubna, Moscow Region, Russia \\ ${ }^{2}$ Institut für Theoretische Physik, Leibniz Universität Hannover, Appelstraße 2, 30167 Hannover, Germany \\ ${ }^{3}$ Riemann Center for Geometry and Physics, Leibniz Universität Hannover, Appelstraße 2, 30167 Hannover, Germany
}

(Received 4 May 2017; revised manuscript received 1 June 2017; published 11 August 2017)

\begin{abstract}
We consider pure $\mathrm{SU}(2)$ Yang-Mills theory on four-dimensional de Sitter space $\mathrm{dS}_{4}$ and construct a smooth and spatially homogeneous magnetic solution to the Yang-Mills equations. Slicing $\mathrm{dS}_{4}$ as $\mathbb{R} \times S^{3}$, via an $\mathrm{SU}(2)$-equivariant ansatz, we reduce the Yang-Mills equations to ordinary matrix differential equations and further to Newtonian dynamics in a double-well potential. Its local maximum yields a Yang-Mills solution whose color-magnetic field at time $\tau \in \mathbb{R}$ is given by $\tilde{B}_{a}=-\frac{1}{2} I_{a} /\left(R^{2} \cosh ^{2} \tau\right)$, where $I_{a}$ for $a=1,2,3$ are the $\mathrm{SU}(2)$ generators and $R$ is the de Sitter radius. At any moment, this spatially homogeneous configuration has finite energy, but its action is also finite and of the value $-\frac{1}{2} j(j+1)(2 j+1) \pi^{3}$ in a spin- $j$ representation. Similarly, the double-well bounce produces a family of homogeneous finite-action electric-magnetic solutions with the same energy. There is a continuum of other solutions whose energy and action extend down to zero.
\end{abstract}

DOI: 10.1103/PhysRevLett.119.061601

Introduction and summary.-Yang-Mills theory with Higgs fields governs three fundamental forces of nature. It has a number of particlelike solutions such as vortices, magnetic monopoles, and instantons [1-3]. In Minkowski space $\mathbb{R}^{3,1}$ smooth vortex and monopole solutions can be constructed only in the presence of Higgs fields. Magnetic monopoles play a key role in the dual superconductor mechanism for the confinement of quarks in QCD based on the condensation of non-Abelian monopoles [4]. However, there are no Higgs fields in QCD, and without them all monopole solutions of pure Yang-Mills theory on $\mathbb{R}^{3,1}$ are singular Abelian monopoles, with the $\mathrm{U}(1)$ gauge group embedded into a higher-dimensional non-Abelian gauge group, e.g., SU(3) for the QCD case. Furthermore, a number of theorems rule out any static, real, finite-energy solution of pure $\mathrm{SU}(2)$ Yang-Mills theory on $\mathbb{R}^{3,1}[5,6]$. Under some mild assumptions the nonexistence can be extended also to time-dependent finite-energy solutions in pure Yang-Mills theory on $\mathbb{R}^{3,1}[7,8]$.

The still elusive quantitative understanding of the confinement mechanism in Minkowski space is not the only problem. In most inflationary models the early and late expansion of the Universe is approximated by a $\mathrm{dS}_{4}$ phase. Therefore, it is important to understand de Sitter vacua of supergravity and string theory [9]. Such vacua include one or more anti-D3-branes [10] (for recent results see, e.g., Ref. [11]). In fact, gauge theories in $\mathrm{dS}_{4}$ occur naturally in string-theoretic constructions from stacks of branes or from compactifications. Hence, constructing explicit solutions and studying their physical effects in gauge theories on de Sitter space are also important for understanding the early Universe and its evolution.

So, on the one hand, Minkowski space does not seem to admit physical non-Abelian field configurations. On the other hand, our Universe appears to be asymptotically de Sitter (not Minkowski) at very early and very large times. This is a strong argument for searching for finite-energy solutions in pure Yang-Mills theory on four-dimensional de Sitter space $\mathrm{dS}_{4}$. The construction of such solutions is the goal of our Letter. [Note that we consider the spacetime background as nondynamical; i.e., we ignore the backreaction on it. The coupled system is governed by the Einstein-Yang-Mills equations (for numerical solutions, see, e.g., the review of Ref. [12] and references therein). However, in this more general setup it is practically impossible to obtain analytic solutions.]

We will not only show that smooth non-Abelian solutions with finite energy indeed exist in pure Yang-Mills theory on $\mathrm{dS}_{4}$, but we will also construct them analytically in a rather simple geometric form. On the spatial $S^{3}$ slices of $\mathrm{dS}_{4}$, the gauge potential as well as the color-electric and colormagnetic fields are constant, analogous to the Dirac monopole on $\mathbb{R}^{3}$ restricted to $S^{2}$ or the Yang monopole on $\mathbb{R}^{5}$ restricted to $S^{4}$ [13]. Furthermore, their temporal variation is such that the action functional is also finite. One may hope that such configurations will help to get a quantitative understanding of QCD confinement in a de Sitter background.

Description of de Sitter space $\mathrm{dS}_{4}$. - Topologically, de Sitter space is $\mathbb{R} \times S^{3}$, and it can be embedded into fivedimensional Minkowski space $\mathbb{R}^{4,1}$ by the help of

$$
\delta_{i j} y^{i} y^{j}-\left(y^{5}\right)^{2}=R^{2}, \quad \text { where } i, j=1, \ldots, 4 .
$$

One can parametrize $\mathrm{dS}_{4}$ with global coordinates $\left(\tau, \theta^{a}\right)$, $a=1,2,3$, by setting (see, e.g., Ref. [14])

$$
y^{i}=R \omega^{i} \cosh \tau, \quad y^{5}=R \sinh \tau \quad \text { with } \quad \tau \in \mathbb{R}
$$


for

$$
\begin{aligned}
& \omega^{1}=\cos \theta_{1}, \quad \omega^{2}=\sin \theta_{1} \cos \theta_{2}, \\
& \omega^{3}=\sin \theta_{1} \sin \theta_{2} \cos \theta_{3}, \\
& \omega^{4}=\sin \theta_{1} \sin \theta_{2} \sin \theta_{3},
\end{aligned}
$$

where $0 \leq \theta_{1}, \theta_{2}<\pi$ and $0 \leq \theta_{3} \leq 2 \pi$. Then, from the flat metric on $\mathbb{R}^{4,1}$ one obtains the induced metric on $\mathrm{dS}_{4}$,

$$
\begin{aligned}
d s^{2} & =R^{2}\left(-d \tau^{2}+\cosh ^{2} \tau \delta_{a b} e^{a} e^{b}\right) \\
& =-d \tilde{\tau}^{2}+R^{2} \cosh ^{2} \frac{\tilde{\tau}}{R} d \Omega_{3}^{2} \quad \text { with } \quad \tilde{\tau}=R \tau,
\end{aligned}
$$

where $d \Omega_{3}^{2}$ is the metric on the unit sphere $S^{3} \cong \mathrm{SU}(2)$ and $\left\{e^{a}\right\}$ is an orthonormal basis of left-invariant 1-forms on $S^{3}$ satisfying

$$
d e^{a}+\varepsilon_{b c}^{a} e^{b} \wedge e^{c}=0 .
$$

We rewrite the metric (2.4) in conformal coordinates $\left(t, \theta^{a}\right)$ by the time reparametrization [14]

$$
\begin{aligned}
t & =\arctan (\sinh \tau)=2 \arctan \left(\tanh \frac{\tau}{2}\right) \Leftrightarrow \frac{d \tau}{d t} \\
& =\cosh \tau=\frac{1}{\cos t},
\end{aligned}
$$

in which $\tau \in(-\infty, \infty)$ corresponds to $t \in(-(\pi / 2)$, $(\pi / 2))$. The metric (2.4) in these coordinates reads

$$
d s^{2}=\frac{R^{2}}{\cos ^{2} t}\left(-d t^{2}+\delta_{a b} e^{a} e^{b}\right)=\frac{R^{2}}{\cos ^{2} t} d s_{\mathrm{cyl}}^{2},
$$

where

$$
d s_{\mathrm{cyl}}^{2}=-d t^{2}+\delta_{a b} e^{a} e^{b}
$$

is the standard metric on the Lorentzian cylinder $\mathbb{R} \times S^{3}$. Hence, four-dimensional de Sitter space is conformally equivalent to the finite cylinder $\mathcal{I} \times S^{3}$ with the metric (2.8), where $\mathcal{I}$ is the interval $(-(\pi / 2),(\pi / 2))$ parametrized by $t$.

Reduction of Yang-Mills to matrix equations and to double-well dynamics.-Since the Yang-Mills equations are conformally invariant, their solutions on de Sitter space can be obtained by solving the equations on $\mathcal{I} \times S^{3}$ with the cylindrical metric (2.8). Therefore, we will consider rank- $N$ vector bundles over this cylinder $\mathcal{I} \times S^{3}$ with the de Sitter metric (2.7) or cylindrical metric (2.8). Our gauge potentials $\mathcal{A}$ and the gauge fields $\mathcal{F}=d \mathcal{A}+\mathcal{A} \wedge \mathcal{A}$ take values in the Lie algebra $s u(N)$. The conformal boundary of $\mathrm{dS}_{4}$ consists of the two three-spheres at $t= \pm(\pi / 2)$ or, equivalently, at $\tau= \pm \infty$. On manifolds $M$ with a nonempty boundary $\partial M$, the group of gauge transformations is naturally restricted to the identity when reaching $\partial M$ (see, e.g., Ref. [15]). This corresponds to a framing of the gauge bundle over the boundary. For our case, this means allowing only gauge-group elements $g(y)$ obeying $g(\partial M)=\operatorname{Id}$ on $\partial M=S_{t= \pm \frac{\pi}{2}}^{3}=S_{\tau= \pm \infty}^{3}$.

In order to obtain explicit solutions we use the SU(2)-equivariant ansatz (cf. Refs. [16-18])

$$
\mathcal{A}(y)=X_{a}(t) e^{a}
$$

for the $s u(N)$-valued gauge potential $\mathcal{A}$ in the temporal gauge $\mathcal{A}_{0} \equiv \mathcal{A}_{t}=0=\mathcal{A}_{\tau}$. Here, $X_{a}(t)$ are three $\operatorname{su}(N)$ valued matrices depending only on $t \in \mathcal{I}$, and $e^{a}$ are basis 1-forms on $S^{3}$ satisfying Eq. (2.5). The corresponding gauge field reads

$$
\begin{aligned}
\mathcal{F} & =\mathcal{F}_{0 a} e^{0} \wedge e^{a}+\frac{1}{2} \mathcal{F}_{b c} e^{b} \wedge e^{c} \\
& =\dot{X}_{a} e^{0} \wedge e^{a}+\frac{1}{2}\left(-2 \varepsilon_{b c}^{a} X_{a}+\left[X_{b}, X_{c}\right]\right) e^{b} \wedge e^{c},
\end{aligned}
$$

where $\dot{X}_{a}:=d X_{a} / d t$ and $e^{0}:=d t$. It is not difficult to show (see, e.g., Ref. [18]) that the Yang-Mills equations on $\mathcal{I} \times S^{3}$ after substituting Eqs. (3.1) and (3.2) reduce to the ordinary matrix differential equations

$$
\ddot{X}_{a}=-4 X_{a}+3 \varepsilon_{a b c}\left[X_{b}, X_{c}\right]-\left[X_{b},\left[X_{a}, X_{b}\right]\right] .
$$

To be more concrete, we let the gauge potential and fields take values in an $s u(2)$ subalgebra of $s u(N)$. In other words, we pick three $\mathrm{SU}(2)$ generators $I_{a}$ in a spin- $j$ representation embedded into $s u(N)$ obeying

$$
\left[I_{b}, I_{c}\right]=2 \varepsilon_{b c}^{a} I_{a} \quad \text { and } \quad \operatorname{tr}\left(I_{a} I_{b}\right)=-4 C(j) \delta_{a b},
$$

where $C(j)=\frac{1}{3} j(j+1)(2 j+1)$

is the second-order Dynkin index of the representation. Explicit solutions to the matrix equations (3.3) can then be found with the natural choice

$$
X_{a}(t)=\frac{1}{2}[1+\psi(t)] I_{a},
$$

where $\psi(t)$ is a real function. The Yang-Mills equations finally boil down to

$$
\begin{aligned}
\ddot{\psi} & =2 \psi(1+\psi)(1-\psi)=-\frac{d V}{d \psi} \\
\text { for } V(\psi) & =\frac{1}{2}\left(1-\psi^{2}\right)^{2} .
\end{aligned}
$$

This is Newton's equation for a particle in a double-well potential, whose solutions are well known. 
The simplest ones are constant at the critical points of $V$, i.e.,

$$
\begin{aligned}
& \psi(t)= \pm 1(\text { minima, } V=0) \quad \text { and } \\
& \psi(t)=0\left(\text { local maximum, } V=\frac{1}{2}\right) .
\end{aligned}
$$

A prominent nontrivial solution is the bounce

$\psi(t)=\sqrt{2} \operatorname{sech}\left(\sqrt{2}\left(t-t_{0}\right)\right)=\frac{\sqrt{2}}{\cosh \left(\sqrt{2}\left(t-t_{0}\right)\right)}$,

which makes an excursion from $\left(\psi=0, V=\frac{1}{2}\right)$ at $t=$ $-\infty$ to $\left(\psi=\sqrt{2}, V=\frac{1}{2}\right)$ at $t=t_{0}$ and back at $t=\infty$. An antibounce is given by $-\psi(t)$. In addition, there is a continuum of periodic solutions oscillating either about $\psi= \pm 1$ or exploring both wells, which are given by Jacobi elliptic functions. Usually, the moduli parameter $t_{0}$ is trivial because of time translation invariance in Eq. (3.6). However, since for de Sitter space according to Eq. (2.6) we consider the solutions $\psi(t)$ only in the interval $\mathcal{I}=(-(\pi / 2),(\pi / 2))$ without imposing boundary conditions, the value of $t_{0} \in \mathbb{R}$ makes a difference. It allows us to pick a segment of length $\pi$ anywhere on the profile of the bounce, not necessarily including its minimum. Finally, we remark that the Newtonian energy conservation produces the relation

$$
\frac{1}{2} \dot{\psi}^{2}=V_{0}-V(\psi)=V_{0}-\frac{1}{2}\left(1-\psi^{2}\right)^{2},
$$

where $V_{0}$ is the value of $V$ at the turning points.

Magnetic and electric-magnetic Yang-Mills configurations on $\mathrm{dS}_{4}$. - Let us look at the gauge potential and field and compute its energy and action in terms of $\psi$. Inserting Eq. (3.5) into Eq. (3.2), we obtain

$$
\begin{aligned}
\mathcal{A} & =\frac{1}{2}(1+\psi) e^{a} I_{a} \text { and } \\
\mathcal{F} & =\left(\frac{1}{2} \dot{\psi} e^{0} \wedge e^{a}-\frac{1}{4}\left(1-\psi^{2}\right) \varepsilon_{b c}^{a} e^{b} \wedge e^{c}\right) I_{a},
\end{aligned}
$$

which yields the color-electric and color-magnetic field components (in the cylinder metric)

$$
\begin{aligned}
E_{a} & =\mathcal{F}_{0 a}=\frac{1}{2} \dot{\psi} I_{a} \quad \text { and } \\
B_{a} & =\frac{1}{2} \varepsilon_{a b c} \mathcal{F}_{b c}=-\frac{1}{2}\left(1-\psi^{2}\right) I_{a} .
\end{aligned}
$$

The electric and magnetic energy densities then become

$$
\begin{aligned}
\rho_{e} & =-\frac{1}{4} \operatorname{tr} E_{a} E_{a}=\frac{3}{4} C(j) \dot{\psi}^{2} \quad \text { and } \\
\rho_{m} & =-\frac{1}{4} \operatorname{tr} B_{a} B_{a}=\frac{3}{4} C(j)\left(1-\psi^{2}\right)^{2},
\end{aligned}
$$

respectively. The energy of our Yang-Mills configuration (in the cylinder metric) computes to

$$
\begin{aligned}
E_{t} & =\int_{S^{3}} e^{1} \wedge e^{2} \wedge e^{3}\left(\rho_{e}+\rho_{m}\right) \\
& =\frac{3}{4} C(j) \operatorname{vol}\left(S^{3}\right)\left(\dot{\psi}^{2}+\left(1-\psi^{2}\right)^{2}\right) \\
& =3 \pi^{2} C(j) V_{0},
\end{aligned}
$$

where we have employed the energy relation (3.9) in the last step. Remarkably, $E_{t}$ is constant and only given by the "double-well energy" $V_{0}$. It is important to note, however, that $E_{t}$ is conjugate to the time variable $t$, and so the energy conjugate to de Sitter time $\tilde{\tau}$ (2.4) is obtained as

$$
\begin{aligned}
E_{\tilde{\tau}} & =\frac{d t}{d \tilde{\tau}} E_{t}=\frac{1}{R} \frac{d t}{d \tau} E_{t}=\frac{1}{R \cosh \tau} E_{t} \\
& =\frac{3 \pi^{2} C(j) V_{0}}{R \cosh \tau} .
\end{aligned}
$$

We see that this energy decays exponentially for early and late times.

In a similar fashion one can evaluate the action functional on the configuration (4.2). Its value is independent of the metric chosen in the computation. For the cylinder metric (2.8), for example, we get

$$
\begin{aligned}
S & =\frac{1}{8} \int_{\mathcal{I} \times S^{3}} e^{0} \wedge e^{1} \wedge e^{2} \wedge e^{3} \operatorname{tr}\left(-2 \mathcal{F}_{0 a} \mathcal{F}_{0 a}+\mathcal{F}_{a b} \mathcal{F}_{a b}\right) \\
& =\int_{\mathcal{I}} d t \operatorname{vol}\left(S^{3}\right)\left(\rho_{e}-\rho_{m}\right) \\
& =\frac{3}{2} \pi^{2} C(j) \int_{-\pi / 2}^{\pi / 2} d t\left(\dot{\psi}^{2}-\left(1-\psi^{2}\right)^{2}\right) \\
& =3 \pi^{3} C(j) V_{0}-6 \pi^{2} C(j) \int_{-\pi / 2}^{\pi / 2} d t V(\psi(t))
\end{aligned}
$$

which takes a finite value for any solution $\psi(t)$ to Eq. (3.6).

For alternatively computing the action directly with the de Sitter metric (2.4), we introduce on orthonormal basis on $\mathrm{dS}_{4}$,

$$
\tilde{e}^{0}:=R d \tau, \quad \tilde{e}^{a}:=R \cosh \tau e^{a},
$$

and expand 
$\mathcal{A}=\tilde{\mathcal{A}}_{a} \tilde{e}^{a} \quad$ and $\quad \mathcal{F}=\tilde{\mathcal{F}}_{0 a} \tilde{e}^{0} \wedge \tilde{e}^{a}+\frac{1}{2} \tilde{\mathcal{F}}_{b c} \tilde{e}^{b} \wedge \tilde{e}^{c}$

so that

$$
\begin{aligned}
\mathcal{A}_{a} & =R \cosh \tau \tilde{\mathcal{A}}_{a}, \quad \mathcal{F}_{b c}=R^{2} \cosh ^{2} \tau \tilde{\mathcal{F}}_{b c}, \\
\mathcal{F}_{0 a} & =\partial_{t} \mathcal{A}_{a}=R^{2} \cosh ^{2} \tau \partial_{\tilde{\tau}} \tilde{\mathcal{A}}_{a} .
\end{aligned}
$$

With these ingredients, we find that

$$
\begin{aligned}
S & =\frac{1}{8} \int_{\mathrm{dS}_{4}} \tilde{e}^{0} \wedge \tilde{e}^{1} \wedge \tilde{e}^{2} \wedge \tilde{e}^{3} \operatorname{tr}\left(-2 \tilde{\mathcal{F}}_{0 a} \tilde{\mathcal{F}}_{0 a}+\tilde{\mathcal{F}}_{a b} \tilde{\mathcal{F}}_{a b}\right) \\
& =\int_{\mathbb{R}} d \tau \operatorname{vol}\left(S^{3}\right) \frac{\rho_{e}-\rho_{m}}{\cosh \tau},
\end{aligned}
$$

which agrees with Eq. (4.6) by virtue of Eq. (2.6) (note that $\int d \tau / \cosh \tau$ also produces the factor of $\pi$ ).

Explicit examples.-Finally, we analytically display the Yang-Mills configurations for the explicit solutions (3.7) and (3.8). The solutions $\psi(t)= \pm 1$ correspond to the vacuum $\mathcal{F}=0$, which is not interesting. In contrast, $\psi(t)=0$ provides the nontrivial smooth configuration [we remark that that $e^{a} I_{a}=g^{-1} d g$, where $g\left(\theta^{a}\right): S^{3} \rightarrow$ $\mathrm{SU}(2)$ is a smooth map of degree (winding number) 1]

$$
\begin{aligned}
& \mathcal{A}=\frac{1}{2} e^{a} I_{a}=\frac{1}{2 R \cosh \tau} \tilde{e}^{a} I_{a}, \\
& \mathcal{F}=-\frac{1}{4} \varepsilon_{b c}^{a} e^{b} \wedge e^{c} I_{a}=-\frac{1}{4 R^{2} \cosh ^{2} \tau} \varepsilon_{b c}^{a} \tilde{e}^{b} \wedge \tilde{e}^{c} I_{a} ;
\end{aligned}
$$

hence (in the de Sitter metric),

$$
\tilde{E}_{a}=0 \quad \text { and } \quad \tilde{B}_{a}=-\frac{I_{a}}{2 R^{2} \cosh ^{2} \tau} .
$$

This is a purely magnetic Yang-Mills field uniform on $S^{3}$ that varies with time $\tau$ and decays exponentially for $\tau \rightarrow \pm \infty$. According to Eq. (4.5) with $V_{0}=1 / 2$, the de Sitter energy of this configuration is finite,

$$
E_{\tilde{\tau}}=\frac{3 \pi^{2} C(j)}{2 R \cosh \tau},
$$

and the action is as well,

$$
S=-\frac{3}{2} \pi^{3} C(j),
$$

gleaned from Eq. (4.6). One may restore the gauge coupling in the denominator of Eq. (5.4).

From the bounce (3.8) we obtain a whole family of nonsingular Yang-Mills configurations,

$$
\begin{aligned}
\mathcal{A}= & \frac{\cos t}{2 R}\left[1+\frac{\sqrt{2}}{\cosh \left(\sqrt{2}\left(t-t_{0}\right)\right)}\right] \tilde{e}^{a} I_{a}, \\
\mathcal{F}= & -\frac{\cos ^{2} t}{4 R^{2}}\left[4 \frac{\sinh \left(\sqrt{2}\left(t-t_{0}\right)\right)}{\cosh ^{2}\left(\sqrt{2}\left(t-t_{0}\right)\right)} \tilde{e}^{0} \wedge \tilde{e}^{a}\right. \\
& \left.+\left(1-\frac{2}{\cosh ^{2}\left(\sqrt{2}\left(t-t_{0}\right)\right)}\right) \varepsilon_{b c}^{a} \tilde{e}^{b} \wedge \tilde{e}^{c}\right] I_{a}
\end{aligned}
$$

depending on $t_{0} \in \mathbb{R}$, where $t=t(\tau)$ via Eq. (2.6) is understood. Clearly, this family carries electric as well as magnetic fields. Since the bounce also has $V_{0}=1 / 2$, the energy of this family coincides with that of the above purely magnetic configuration, given by Eq. (5.3). Its action, however, is different: from Eq. (4.6) we find

$$
\begin{aligned}
\frac{S}{C(j)}= & -\frac{3}{2} \pi^{3}+12 \pi^{2} \int_{-\pi / 2}^{\pi / 2} d t \frac{\sinh ^{2}\left(\sqrt{2}\left(t-t_{0}\right)\right)}{\cosh ^{4}\left(\sqrt{2}\left(t-t_{0}\right)\right)} \\
= & -\frac{3}{2} \pi^{3}+\sqrt{8} \pi^{2}\left[\tanh ^{3}\left(\frac{\pi}{\sqrt{2}}+\delta\right)\right. \\
& \left.+\tanh ^{3}\left(\frac{\pi}{\sqrt{2}}-\delta\right)\right],
\end{aligned}
$$

where $\delta=\sqrt{2} t_{0} \in \mathbb{R}$. Its numerical value varies between 5.52 (for $\delta=0$ ) and -46.51 (for $\delta \rightarrow \pm \infty$ ).

In fact, for any choice of turning point and time, $V_{0}=$ $V\left(\psi_{0}\right)$ and $\psi_{0}=\psi\left(t_{0}\right)$, there is a unique solution $\psi(t)$ that gives rise to a smooth and $S^{3}$-homogeneous Yang-Mills solution $\mathcal{F}$ with both color-electric and color-magnetic fields present. All their energies and actions remain finite. As a final example, consider small oscillations about the vacuum $\psi=1$ in the harmonic approximation,

$\psi(t)=1+A \cos \left(2\left(t-t_{0}\right)\right) \Rightarrow V_{0}=-\frac{1}{2}+2 A^{2}$.

Neglecting terms of order $A^{3}$, it is easily calculated that in this case, independent of $t_{0}$,

$\rho_{e}=\rho_{m}=\frac{3}{4} C(j) A^{2} \Rightarrow E_{t}=6 \pi^{2} C(j) A^{2} \quad$ and $\quad S=0$.

In summary, we have described a class of classical pure Yang-Mills configurations (without Higgs fields) on de Sitter space $\mathrm{dS}_{4}$ that are spatially homogeneous and decay for early and late times. Their energies and actions are all finite. Therefore, the described gauge configurations can be important in a semiclassical analysis of the path integral for quantum Yang-Mills theory on $\mathrm{dS}_{4}$. These Yang-Mills solutions may help in understanding the dual superconducting mechanism of confinement on de Sitter space. 
This work was partially supported by Deutsche Forschungsgemeinschaft Grant No. LE 838/13 and by the Heisenberg-Landau program.

*ita@theor.jinr.ru

†olaf.lechtenfeld@itp.uni-hannover.de

*alexander.popov@itp.uni-hannover.de

[1] A. Actor, Rev. Mod. Phys. 51 (1979) 461.

[2] R. Rajaraman, Solitons and Instantons (North-Holland, Amsterdam, 1984).

[3] N. Manton and P. Sutcliffe, Topological Solitons (Cambridge University Press, Cambridge, England, 2004).

[4] G. 't Hooft, Gauge fields with unified weak, electromagnetic, and strong interactions, Conference: C75-06-23 (Palermo Conf.1975:1225), PRINT-75-0836 (UTRECHT); S. Mandelstam, Phys. Lett. 53B, 476 (1975); Phys. Rep. 23, 245 (1976).

[5] S. R. Coleman, Secret symmetry: An introduction to spontaneous symmetry breakdown and gauge fields, Conference: C73-06-05 (Erice Subnucl.Phys.1973:139), Subnucl. Ser. Vol. 11 (1975) p. 139.

[6] S. Deser, Phys. Lett. 64B, 463 (1976).
[7] S. R. Coleman, Commun. Math. Phys. 55, 113 (1977).

[8] M. Magg, J. Math. Phys. (N.Y.) 19, 991 (1978).

[9] S. Kachru, R. Kallosh, A. Linde, and S. P. Trivedi, Phys. Rev. D 68, 046005 (2003).

[10] S. Kachru, R. Kallosh, A. Linde, J. Maldacena, L. McAllister, and S. P. Trivedi, J. Cosmol. Astropart. Phys. 10 (2003) 013.

[11] E. A. Bergshoeff, K. Dasgupta, R. Kallosh, A. Van Proeyen, and T. Wrase, J. High Energy Phys. 05 (2015) 058.

[12] B. Kleihaus, J. Kunz, and F. Navarro-Lerida, Classical Quantum Gravity 33, 234002 (2016).

[13] C. N. Yang, J. Math. Phys. (N.Y.) 19, 320 (1978).

[14] S. W. Hawking and G. F. R. Ellis, The Large Scale Structure of Space-time (Cambridge University Press, Cambridge, England, 1975).

[15] S. K. Donaldson, J. Geom. Phys. 8, 89 (1992).

[16] T. A. Ivanova and O. Lechtenfeld, Phys. Lett. B 670, 91 (2008).

[17] T. A. Ivanova, O. Lechtenfeld, A. D. Popov, and T. Rahn, Lett. Math. Phys. 89, 231 (2009).

[18] I. Bauer, T. A. Ivanova, O. Lechtenfeld, and F. Lubbe, J. High Energy Phys. 10 (2010) 044. 